\title{
The gut microbiota in the elderly
}

\author{
Karin Šik Novak, Zala Jenko Pražnikar \\ Faculty of Health Sciences, University of Primorska, Izola, Slovenia \\ karin.novak@fvz.upr.si, zala.praznikar@fvz.upr.si
}

\section{Abstract}

Introduction: The human gastrointestinal tract represents the biggest microbial community in the human body. Gut bacteria regulate extraction, synthesis and absorption of many nutrients and metabolites, such as bile acids, lipids, amino acids, vitamins, and short-chain fatty acids. Aging is a genetically-determined process that leads to a decline of physiological functions. One of the most recognized effects of aging is the age-associated immune deregulation. Gut dysbiosis can trigger the immune response and chronic low-grade inflammation, leading to age-related degenerative pathologies and unhealthy aging. An adequate nutritional status is important to maintain the functionality of the immune system and preventing frailty and cognitive function impairment in the elderly. Methods: A scientific literature search was performed by accessing the databases PubMed, GoogleScholar and ScienceDirect using the following keywords and their combinations: "gut microbiota«, »elderly«, »longevity«, »Alzheimer's disease«, "nutrition", limiting the search to the last 10 years. The review was focused on the changes of gut microbiota during aging, the effect of nutrition and its link to pathologies. Results: Gut microbiota diversity increases from birth and at about the age of three, the gut microbiota composition is mostly mature. In the elderly, changes in dietary habits, digestion, nutrient absorption and immune activity can affect gut microbiota composition. The microbiota-gut-brain axis is a bidirectional communication system that is connected via neural, immune, endocrine, and metabolic pathways and plays a key role in the modulation of brain functions. Research shows a link between the gut microbiota and Alzheimer's disease (AD), which is characterized by the accumulation of amyloid- $\beta$ and tau in the brain, leading to cognitive impairment. The increased gut and blood-brain barrier permeability induced by gut dysbiosis may affect pathogenesis of $\mathrm{AD}$ and other neurodegenerative 
disorders. Nutritional strategies aiming at microbiota and immune modulation in the elderly are especially relevant due to their higher susceptibility to disease, malnutrition, impairment of the gut microbiota and a pro-inflammatory status of the immune system. Animal studies show that interventions with antibiotics, microbial infections, prebiotics, probiotics and faecal microbiota transplantation not only affect gut microbiota composition, but also influence cognitive impairment related to AD. Analysis of the gut microbiota and its potential for modulation to improve health are important elements in the process of improving health of the elderly. Discussion and conclusions: Nutritional strategies for the elderly should consider the nutritional deficiencies and needs, but also the gut microbiota, the immune system and cognitive function. More research is needed to better understand the changes in gut microbiota over the lifespan and associated pathologies.

Keywords: gut microbiota, aging, elderly, intestine, nutrition

\section{Introduction}

The human gastrointestinal tract contains more than $10^{12}$ of microorganisms per millilitre and represents the biggest microbial community in the human body. Gut bacteria are key regulators of extraction, synthesis and absorption of many nutrients and metabolites, such as bile acids, lipids, amino acids, vitamins and short-chain fatty acids (SCFA) (Rinninella et al., 2019). Gut microbiota can affect distant organs by regulating the absorption of nutrients or the production of microbial metabolites such as SCFA or vitamins, by regulating the immune system and by translocating bacteria or their products through disrupted mucosal barriers (Shimizu, 2018). For the last two centuries the lifespan has been increasing by two years per decade in the developed countries (Salazar et al., 2017). Most western countries have experienced a significant increase in the proportion of elderly citizens (65 years and older) due to improved sanitation, hygiene and healthcare (O'Toole and Jeffery, 2015). One of the most recognized effects of aging is the age-associated immune deregulation (Salazar et al., 2017). Gut dysbiosis can trigger the innate immune response and chronic low-grade inflammation, leading to many age-related degenerative pathologies and unhealthy aging (Kim and Jazwinski, 2018). An adequate nutritional status is important to maintain the functionality of the immune system and preventing frailty and cognitive function in the elderly (Salazar et al., 2017). Analysis of the gut microbiota and its potential for modulation to improve health are important elements in the process of improving health of the elderly (O'Toole and Jeffery, 2015).

\section{Methods}

We performed a literature search accessing the databases PubMed, GoogleScholar and ScienceDirect using following keywords and their combinations: »gut microbiota«, »gut microbiome«, »elderly«, »longevity«, »Alzheimer’s dis- 
ease«. We limited the search to the last 10 years. The literature review was focused on the change of gut microbiota during aging, the effect of nutrition on gut microbiota and various nutritional and other interventions for microbiota manipulation and its link to neurodegenerative diseases and pathologies.

\section{Results and Discussion}

Gut microbiota shows wide inter-individual variation, but its within-individual variation is relatively stable over time (Kim and Jazwinski, 2018). Taxonomically, bacteria are classified according to phyla, classes, orders, families, genera and species (Rinninella et al., 2019). The dominant bacterial phyla are Bacteroidetes and Firmicutes, which constitute $90 \%$ of the total gut microbiota (Kim \& Jazwinski, 2018), with Actinobacteria, Proteobacteria, Fusobacteria, and Verrucomicrobia also frequently present. Microbiota diversity increases from birth and at about the age of three years, a child's gut microbiota composition is mostly adult-like (Rinninella et al., 2019). The composition of the intestinal microbiota in older people is variable between individuals and differs from the microbiota and diversity levels of younger adults (Claesson et al., 2012). In the elderly, changes in dietary habits, digestion, nutrient absorption and weaker immune activity can affect gut microbiota composition (Rinninella et al., 2019). Changes in diet could be attributed to the loss of taste and smell and difficulties with chewing food. This frequently leads to the increased intake of foods high in fat and sugar and decreased intake of plant foods (Mangiola et al., 2018). The loss of diversity in the common microbiota groups is associated with increased frailty and reduced cognitive performance (O'Toole and Jeffery, 2015).

The microbiota-gut-brain axis is a bidirectional communication system that is connected through neural, immune, endocrine and metabolic pathways. The gut microbiota plays a vital role in the modulation of brain functions (Jiang et al., 2017). Gut bacteria have been found to affect the maturation of the nervous, endocrine and immune systems in mice (Pluta et al., 2020). Research supports a possible link between the gut microbiota and Alzheimer's disease (AD), which is characterized by the deposition of amyloid- $\beta$ (A $\beta)$ and tau in the brain, leading to degeneration of cognitive function (Mangiola et al., 2018). Theories for the role of gut microbiota in AD pathophysiology include direct microbial action (microbial infection in AD), indirect action (hygene hypothesis) and processes related to aging of the immune system (Angelucci et al., 2019). The increased gut and blood-brain barrier permeability induced by microbiota dysbiosis may modulate AD pathogenesis and other neurodegenerative disorders, especially aging-related (Jiang et al., 2017). The inflammatory response from the gut along with aging and poor diet in the elderly have been found to contribute to the pathogenesis of AD (Pluta et al., 2020). Gut microbiota products, such as SCFA (butyrate, acetate, propionate), have specific functions in the gut and the brain where butyrate has been found to have protective properties in the brain. Gut microbiota also produces more than $90 \%$ of 
whole body serotonin, a metabolite that regulates cognitive functions (Shimi$\mathrm{zu}, 2018)$. Studies show reduced microbial diversity and a decrease in Firmicutes and Bifidobacterium, as well as an increase in Bacteroidetes and Proteobacteria in AD patients (Pluta et al., 2020; Rinninella et al., 2019). Changes in gut microbial composition and diversity are associated with biological or functional age. Gut microbial diversity inversely correlates with biological age, but not with chronological age. Transfer of the gut microbiota from old to young germ-free mice triggers inflammatory responses mimicking "inflammaging" (Kim and Jazwinski, 2018).

Unhealthy dietary patterns, drugs, reactive oxygen species (ROS), psychological stress and other pro-inflammatory factors contribute to gut dysbiosis and are linked to development of various pathologies. Gut dysbiosis due to antibiotics or a high-fat or high-carbohydrate diet is associated with metabolic disorders (Kim and Jazwinski, 2018). Diet is a controllable environmental factor that influences gut microbiota composition (Claesson et al., 2012) and has been shown to be involved in the development of AD (Pluta et al., 2020). Nutritional strategies aiming at microbiota and immune modulation in the elderly are especially relevant due to their higher susceptibility to disease, malnutrition, impairment of the gut microbiota and a pro-inflammatory status of the immune system (Salazar et al., 2017).

The Mediterranean diet (MD) involves a high intake of legumes, olive oil, fruits, nuts, vegetables and whole grains, moderate intake of fish, wine and dairy and a low intake of red meat, processed meat and sugar. Greater adherence to MD was associated with a reduced risk of developing AD (Botchway et al., 2018). Healthy dietary patterns with high probiotic and prebiotic intake, along with other nutrients, may delay cognitive decline (Angelucci et al., 2019). Probiotics are bacteria that are beneficial for host's health if consumed in adequate quantities, while prebiotics are fibre substances that act as food for these bacteria. The consumption of fermented milk product with probiotics not only affected normal brain activity, but also caused significant cognitive improvement in patients with AD (Pluta et al., 2020). A two week dietary intervention in elderly women with obesity improved the metabolic profile and reversed gut microbiota dysbiosis towards a profile associated with metabolic health. A combined use of balanced Mediterranean diet and probiotics could induce beneficial effects on the gut microbiota and metabolic profile (Cancello et al., 2019). The role of prebiotics and diet in modulating AD will need to be established more in the future (Leblhuber et al., 2018). In healthy older adults, probiotics promote mental flexibility and alleviate stress, along with changes in gut microbiota (Kim et al., 2021). An intervention with probiotic milk positively affected cognitive function and some metabolic markers (Akbari et al., 2016) and a multispecies probiotic influenced gut microbiota composition and tryptophan metabolism in AD patients (Leblhuber et al., 2018). A recent systematic review and meta-analysis showed that probiotics intervention improved general cognitive function, inflammatory profile, cellular biomarkers regulation 
and led to significantly increased brain-derived neurotrophic factor (BDNF) levels in humans, while in animals they improved cognitive status and spatial and non-spatial memory. The most widely used probiotic strains were from the genera Lactobacillus and Bifidobacterium, the most commonly used species were L. acidophilus, B. bifidum and B. longum (Ruiz-Gonzalez et al., 2021).

In addition to probiotic interventions, studies with germ-free animals, antibiotic interventions, microbial infections and faecal microbiota transplantation have been used to determine the role of the gut microbiota on cognition and AD. Studies on germ-free mice and disruption of the gut microbiota after antibiotic treatment in humans induce cognitive impairment and decreased BDNF levels in the brain (Jiang et al., 2017). Transfer and transplantation of the faecal microbiota from healthy mice into mice with AD ameliorated the formation of $A \beta$ plaques and cognitive impairment and reversed abnormalities in the colonic expression of genes related to intestinal macrophage activity (Kim et al., 2020). It's important to consider the intervention targets on the intestinal microbiota and the immune system in humans may differ among elderly groups from different environments or geographical locations (Salazar et al., 2017). Despite interventions with probiotics, other strategies have been largely unstudied in humans (Ruiz-Gonzalez et al., 2021).

\section{Conclusions}

Nutritional strategies for the elderly should consider the nutritional deficiencies and needs that differ from the needs in the adult life, but also the gut microbiota, the immune system and cognitive function. Gut microbiota profiling offers the potential for biomarker-based identification of individuals at risk for unhealthy aging and could be used as diagnostic criteria for AD. More research is still needed to better understand the changes in gut microbiota over the lifespan, its link to dietary changes and associated pathologies and to clarify whether a stable gut microbiota modification could be obtained and its longterm effects on frailty and cognitive decline.

\section{References}

AKBARI, E., ASEMI, Z., DANESHVAR KAKHAKI, R., Bahmani, F., Kouchaki, E., Tamtaji, O. R., Hamidi, G. A. and Salami, M., 2016. Effect of Probiotic Supplementation on Cognitive Function and Metabolic Status in Alzheimer's Disease: A Randomized, Double-Blind and Controlled Trial. Frontiers in Aging Neuroscience, vol. 8, pp. 256. https://doi.org/10.3389/ fnagi.2016.00256

Angelucci, F., Cechova, K., Amlerova, J. and Hort, J., 2019. Antibiotics, gut microbiota, and Alzheimer's disease. Journal of Neuroinflammation, vol. 16, no. 1, pp. 108. https://doi.org/10.1186/s12974-019-1494-4

Botchway, B. O. A., Moore, M. K., Akinleye, F. O., Iyer, I. C. and Fang, M., 2018. Nutrition: Review on the Possible Treatment for Alzheimer's Disease. 
Journal of Alzheimer's Disease: JAD, vol. 61, no. 3, pp. 867-883. https:// doi.org/10.3233/JAD-170874

Cancello, R., Turroni, S., Rampelli, S., Cattaldo, S., Candela, M., Cattani, L., Mai, S., Vietti, R., Scacchi, M., Brigidi, P. and Invitti, C., 2019. Effect of Short-Term Dietary Intervention and Probiotic Mix Supplementation on the Gut Microbiota of Elderly Obese Women. Nutrients, vol. 11, no. 12, pp. 3011. https://doi.org/10.339o/nu11123011

Claesson, M. J., Jeffery, I. B., Conde, S., Power, S. E., O’Connor, E. M., Cusack, S., Harris, H. M. B., Coakley, M., Lakshminarayanan, B., O’Sullivan, O., Fitzgerald, G. F., Deane, J., O’Connor, M., Harnedy, N., O’Connor, K., O’Mahony, D., van Sinderen, D., Wallace, M., Brennan, L., ... and O’Toole, P. W., 2012. Gut microbiota composition correlates with diet and health in the elderly. Nature, vol. 488, no. 7410, pp. 178-184. https://doi. org/10.1038/nature11319

Jiang, C., Li, G., Huang, P., Liu, Z. and Zhao, B., 2017. The Gut Microbiota and Alzheimer's Disease. Journal of Alzheimer's Disease, vol. 58, no. 1, pp. 1-15. https://doi.org/10.3233/JAD-161141

Kim, C.-S., Cha, L., Sim, M., Jung, S., Chun, W. Y., Baik, H. W. and Shin, D.-M., 2021. Probiotic Supplementation Improves Cognitive Function and Mood with Changes in Gut Microbiota in Community-Dwelling Older Adults: A Randomized, Double-Blind, Placebo-Controlled, Multicenter Trial. The Journals of Gerontology. Series A, Biological Sciences and Medical Sciences, vol. 76, no. 1, pp. 32-40. https://doi.org/10.1093/gerona/glaao9o

Kim, M.-S., Kim, Y., Choi, H., Kim, W., Park, S., Lee, D., Kim, D. K., Kim, H. J., Choi, H., Hyun, D.-W., Lee, J.-Y., Choi, E. Y., Lee, D.-S., Bae, J.-W. and Mook-Jung, I., 2020. Transfer of a healthy microbiota reduces amyloid and tau pathology in an Alzheimer's disease animal model. Gut, vol. 69, no. 2, pp. 283-294. https://doi.org/10.1136/gutjnl-2018-317431

Kim, S. and Jazwinski, S. M., 2018. The Gut Microbiota and Healthy Aging: A Mini-Review. Gerontology, vol. 64, no. 6, pp. 513-520. https://doi. org/10.1159/000490615

Leblhuber, F., Steiner, K., Schuetz, B., Fuchs, D. and Gostner, J. M., 2018. Probiotic Supplementation in Patients with Alzheimer's Dementia-An Explorative Intervention Study. Current Alzheimer Research, vol. 15, no. 12, pp. 1106-1113. https://doi.org/10.2174/1389200219666180813144834

Mangiola, F., Nicoletti, A., Gasbarrini, A. and Ponziani, F. R., 2018. Gut microbiota and aging. European Review for Medical and Pharmacological Sciences, vol. 22, no. 21, pp. 7404-7413. https://doi.org/10.26355/eurrev_201811_16280

O’Toole, P. W. and Jeffery, I. B., 2015. Gut microbiota and aging. Science, vol. 350, no. 6265, pp. 1214-1215. https://doi.org/10.1126/science.aac8469 
Pluta, R., Ułamek-Kozioł, M., Januszewski, S. and Czuczwar, S. J., 2020. Gut microbiota and pro/prebiotics in Alzheimer's disease. Aging, vol. 12, no. 6, pp. 5539-5550. https://doi.org/10.18632/aging.102930

Rinninella, E., Raoul, P., Cintoni, M., Franceschi, F., Miggiano, G. A. D., Gasbarrini, A. and Mele, M. C., 2019. What is the Healthy Gut Microbiota Composition? A Changing Ecosystem across Age, Environment, Diet, and Diseases. Microorganisms, vol. 7, no. 1. https://doi.org/10.339o/microorganisms7010014

Ruiz-Gonzalez, C., Roman, P., Rueda-Ruzafa, L., Rodriguez-Arrastia, M. and Cardona, D., 2021. Effects of probiotics supplementation on dementia and cognitive impairment: A systematic review and meta-analysis of preclinical and clinical studies. Progress in Neuro-Psychopharmacology \& Biological Psychiatry, vol. 108, pp. 110-189. https://doi.org/10.1016/j.pnpbp.2020.110189

Salazar, N., Valdés-Varela, L., González, S., Gueimonde, M. and Reyes-Gavilán, C. G. de los., 2017. Nutrition and the gut microbiome in the elderly. Gut Microbes, vol. 8, no. 2, pp. 82-97. https://doi.org/10.1080/19490976.2016.1 256525

Shimizu, Y., 2018. Gut microbiota in common elderly diseases affecting activities of daily living. World Journal of Gastroenterology, vol. 24, no. 42, pp. 4750-4758. https://doi.org/10.3748/wjg.v24.i42.4750 